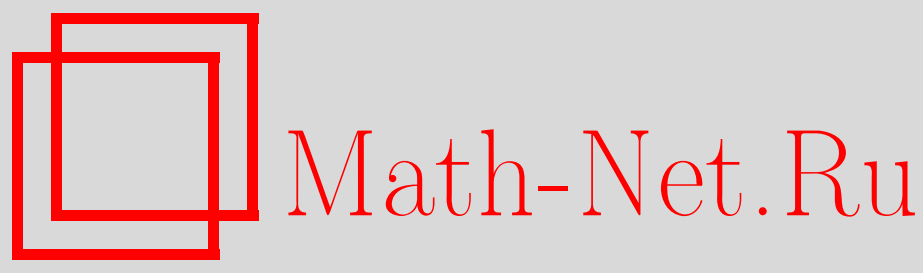

И. А. Тайманов, С. П. Царев, Двумерные рациональные солитоны, построенные с помощью преобразований Мутара, и их распад, ТМФ, 2008, том 157, номер 2, 188-207

DOI: https://doi.org/10.4213/tmf6274

Использование Общероссийского математического портала Math-Net.Ru подразумевает, что вы прочитали и согласны с пользовательским соглашением http://www.mathnet.ru/rus/agreement

Параметры загрузки:

IP : 3.85 .73 .92

26 апреля 2023 г., 10:34:24

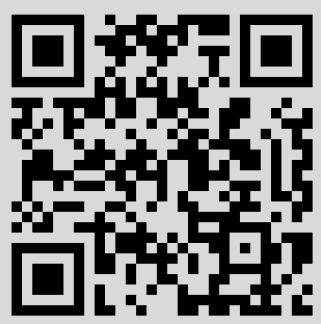




\section{ДВУМЕРНЫЕ РАЦИОНАЛЬНЫЕ СОЛИТОНЫ, ПОСТРОЕННЫЕ С ПОМОЩЬЮ ПРЕОБРАЗОВАНИЙ МУТАРА, И ИХ РАСПАД}

Построено семейство двумерных стационарных операторов Шредингера с быстроубывающими гладкими рациональными потенциалами и нетривиальными $L_{2}$-ядрами. Показано, что некоторые из построенных гладких потенциалов порождают решения уравнения Веселова-Новикова, быстро убывающие на бесконечности, несингулярные при $t=0$ и имеющие особенности при конечных временах $t \geqslant t_{0}>0$.

Ключевые слова: двумерный оператор Шредингера, преобразование Мутара, уравнение Веселова-Новикова, распад решения.

\section{1. ВВЕДЕНИЕ}

В настоящей работе мы используем преобразование Мутара [1], являющееся двумерным обобщением хорошо известного в теории солитонов преобразования Дарбу, в исследовании некоторых проблем спектральной теории двумерных операторов и $(2+1)$-мерных нелинейных эволюционных уравнений. В частности, основные результаты работы состоят в явной конструкции:

1) двумерных операторов Шредингера

$$
H=-\Delta+u=-\left(\partial_{x}^{2}+\partial_{y}^{2}\right)+u(x, y)
$$

с быстроубывающими гладкими рациональными потенциалами и нетривиальными $L_{2}$-ядрами, содержащими по крайней мере двумерное подпространство, порожденное рациональными собственными функциями (см. теоремы 2,3 );

2) распадающихся за конечное время решений уравнения Веселова-Новикова $(\mathrm{BH})$ - двумерного обобщения уравнения Кортевега-де Фриза (КдФ). Упомянутые

* Институт математики им. С. Л. Соболева СО РАН, Новосибирск, Россия.

E-mail: taimanov@math.nsc.ru

${ }^{\dagger}$ Красноярский государственный педагогический университет им. В.П. Астафьева, Красноярск, Россия. E-mail: sptsarev@mail.ru 
решения обладают быстроубывающими гладкими рациональными начальными данными Коши (см. теорему 4).

Первая из вышеуказанных конструкций была анонсирована и кратко описана в работе [2]. Для операторов с потенциалами, убывающими достаточно быстро, существует развитая спектральная теория [3], [4]. Заметим, что для одномерных операторов Шредингера с потенциалами, убывающими достаточно быстро, существование квадратично-суммируемых собственных функций на нулевом уровне энергии невозможно (см., например, [3]). Для операторов в пространствах большой размерности (т.е. при размерности, большей или равной 5) можно легко построить подобные примеры с ядром оператора, содержащим сглаженную функцию Грина оператора $\Delta$. Однако для двумерных операторов построенные нами примеры являются первыми известными примерами операторов, имеющих нетривиальное $L_{2}$-ядро.

Уравнение ВН имеет вид

$$
\begin{gathered}
U_{t}=\partial^{3} U+\bar{\partial}^{3} U+3 \partial(U V)+3 \bar{\partial}(\bar{V} U)=0, \\
\bar{\partial} V=\partial U
\end{gathered}
$$

и является первым в иерархии уравнений вида

$$
H_{t}=H A+B H,
$$

где $A$ и $B$ - дифференциальные операторы. Здесь и всюду ниже мы используем стандартные операторы производных $\partial=\partial_{z}=\left(\partial_{x}-i \partial_{y}\right) / 2, \bar{\partial}=\partial_{\bar{z}}=\left(\partial_{x}+i \partial_{y}\right) / 2$ по комплексной переменной $z=x+i y$. Указанные уравнения были введены в работе [5] как уравнения, сохраняющие спектр оператора $H$ на нулевом уровне энергии. Главная часть $n$-го уравнения иерархии ВН имеет вид

$$
U_{t}=\partial^{2 n+1} U+\bar{\partial}^{2 n+1} U+\cdots,
$$

где многоточие обозначает слагаемые меньшего порядка.

Из метода обратной задачи вытекает, что решения уравнения КдФ с аналитическими быстроубывающими данными Коши не распадаются за конечное время. Корректность постановки задачи Коши для этого уравнения доказана для многих функциональных пространств (см. работу [6] и приведенные в ней ссылки). Как показано в данной работе, это неверно для рассматриваемого нами естественного двумерного обобщения КдФ.

Примеры распадающихся решений получены не с помощью метода обратной задачи, который недостаточно развит в рассматриваемом случае. Обратная задача рассеяния для двумерного оператора Шредингера на заданном уровне энергии впервые была поставлена в [7] и изучалась для положительных уровней энергии [8] или уровней ниже основного состояния [9]. Мы полагаем, что изучение этой задачи на нулевом уровне энергии будет весьма полезным для понимания явлений, обсуждаемых в данной работе. 
Следует заметить, что построенные нами потенциалы, полученные итерациями преобразования Мутара, можно рассматривать как двумерное обобщение одномерных рациональных солитонов, полученных аналогичным способом с помощью преобразования Дарбу (см. п. 2.2). Однако в одномерном случае такие потенциалы всегда сингулярны.

Отметим, что потенциалы получены с помощью итераций преобразования Мутара из потенциала, равного постоянной. Эти потенциалы интегрируемы на нулевом уровне энергии в том смысле, что все решения соответствующего уравнения $H \psi=0$ могут быть явно найдены с помощью квадратур и линейных комбинаций гармонических функций. Подробнее указанная конструкция приведена в разделе 5.

\section{2. ПРЕОБРАЗОВАНИЯ ДАРБУ И МУТАРА}

2.1. Преобразование Дарбу. Пусть

$$
H=-\frac{d^{2}}{d x^{2}}+u(x)
$$

является одномерным оператором Шредингера и функция $\omega$ удовлетворяет уравнению $H \omega=0$. Функция $\omega$ определяет факторизацию $H$ :

$$
H=A^{\mathrm{T}} A, \quad A=-\frac{d}{d x}+v, \quad A^{\mathrm{T}}=\frac{d}{d x}+v, \quad v=\frac{\omega_{x}}{\omega} .
$$

Действительно,

$$
A^{\mathrm{T}} A=\left(\frac{d}{d x}+v\right)\left(-\frac{d}{d x}+v\right)=-\frac{d^{2}}{d x^{2}}+v^{2}+v_{x}
$$

и уравнение

$$
v_{x}+v^{2}=u
$$

тем самым эквивалентно $H \omega=0$. Если функция $v$ вещественнозначна, то имеет место тождество $A^{*}=A^{\mathrm{T}}$.

Преобразование Дарбу оператора $H$ состоит в перестановке операторов $A^{\mathrm{T}}$ и $A[10]$ :

$$
H=A^{\mathrm{T}} A \rightarrow \widetilde{H}=A A^{\mathrm{T}},
$$

или в терминах $u$ :

$$
u=v^{2}+v_{x} \rightarrow \tilde{u}=v^{2}-v_{x} .
$$

Легко проверить следующее утверждение.

УтвЕРЖДЕНИЕ 1. Если функиия $\varphi$ удовлетворяет уравнению $H \varphi=E \varphi$, где $E=$ const, то функиия $\widetilde{\varphi}=A \varphi$ удовлетворяет уравнению $\widetilde{H} \widetilde{\varphi}=E \widetilde{\varphi}$.

ЗАмечание 1. Вообще говоря, преобразование Дарбу определяется любым решением уравнения $H \omega=c \omega$ при $c=$ const. В этом случае оно сводится к преобразованию оператора $H^{\prime}=H-c$, для которого $H^{\prime} \omega=0$. 


\section{2. Одномерные солитоны, получаемые с помощью преобразования} Дарбу. Пусть $u=0$ и $\omega=\omega_{1}=x=\tau_{1}$. Тогда

$$
v=\frac{1}{x}, \quad v_{x}=-\frac{1}{x^{2}}, \quad u_{1}=\tilde{u}=\frac{2}{x^{2}} .
$$

Функция

$$
\psi(P, x)=\left(1-\frac{1}{i \sqrt{E} x}\right) e^{i \sqrt{E} x}
$$

мероморфна по переменной $P=(E, \lambda)$ на римановой поверхности $\Gamma=\left\{\lambda^{2}=E\right\}$, и для любого значения $E$ ее ветви дают базис решений уравнения

$$
H_{1} \psi=\left(-\frac{d^{2}}{d x^{2}}+u_{1}\right) \psi=E \psi
$$

(мы будем нормировать функцию $\psi$ условием $\psi \approx e^{i \sqrt{E} x}$ при $E \rightarrow \infty$ ). Применяя преобразование Дарбу, определенное $\omega_{2}=x^{2}+\tau_{2} / \omega_{1}$, получаем новый потенциал. В частности, для любого $n$ потенциал

$$
U_{n}=\frac{n(n+1)}{x^{2}}
$$

может быть получен после $n$ итераций. Орбита потенциала $U_{n}$ под действием уравнений иерархии КдФ дает $n$-мерное семейство $M_{n}$ потенциалов.

Справедливы следующие утверждения [11], [12].

1. Существует общая рекуррентная процедура, найденная Адлером и Мозером [11] для определения полиномов $\theta_{n}\left(\tau_{1}, \ldots, \tau_{n}\right)$ таких, что:

а) $\theta_{n}-$ полином степени $n(n+1) / 2$ по переменной $x=\tau_{1}$, в частности

$$
\theta_{1}=x=\tau_{1}, \quad \theta_{2}=x^{3}+\tau_{2}, \quad \theta_{3}=x^{6}+5 \tau_{2} x^{3}+\tau_{3} x-5 \tau_{2}^{2} ;
$$

б) функция

$$
u_{n}(x)=-2 \frac{d^{2}}{d x^{2}} \ln \theta_{n}\left(\tau_{1}+x, \tau_{2}, \ldots, \tau_{n}\right)
$$

является потенциалом $H$, полученным после $n$ итераций преобразования Дарбу (при исходном значении $\left.u_{0}=0\right)$, где $\tau_{k}, k=2,3, \ldots,-$ свободные скалярные параметры (константы интегрирования), появляющиеся последовательно на каждом шаге итерации;

в) для $\varphi_{n}=\theta_{n+1} / \theta_{n}$ имеем

$$
\left(-\frac{d^{2}}{d x^{2}}+u_{n}\right) \varphi_{n}=0 .
$$

2. $M_{n}-n$-мерное семейство, параметризованное $\tau \in \mathbb{C}^{n}$ и состоящее из потенциалов $u_{n}\left(\tau_{1}+x, \tau_{2}, \ldots, \tau_{n}\right)$.

3. Существуют бирациональные преобразования $\tau \rightarrow t$ вида

$$
t_{k}=a_{k} \tau_{k}+g_{k}\left(\tau_{1}, \ldots, \tau_{k-1}\right), \quad a_{k} \neq 0,
$$


такие, что

$$
\frac{\partial u_{n}}{\partial t_{k}}=X_{k}\left(u_{n}\right)
$$

есть $k$-й поток иерархии КдФ.

4. Нули $x_{1}, \ldots, x_{n(n+1) / 2}$ полиномов $\theta_{n}$ при эволюции в соответствии с уравнениями иерархии КдФ определяют некоторые интегрируемые гамильтоновы системы. Например, для исходного уравнения КдФ

$$
u_{t}=3 u u_{x}-\frac{1}{2} u_{x x x}=X_{2}(u)
$$

их динамика описывается системой Калоджеро-Мозера.

2.3. Преобразование Мутара. Пусть $H$ - двумерный потенциальный оператор Шредингера, $\omega$ - решение уравнения

$$
H \omega=(-\Delta+u) \omega=0
$$

Тогда (эллиптическое) преобразование Myтара оператора $H$ (ср. [1]) определено следующим образом:

$$
\widetilde{H}=-\Delta+u-2 \Delta \ln \omega=-\Delta-u+2 \frac{\omega_{x}^{2}+\omega_{y}^{2}}{\omega^{2}} .
$$

УтВеРЖДЕНИЕ 2. Если функиия $\varphi$ удовлетворяет уравнению $H \varphi=0$, то функиия $\theta$, определяемая из совместной системы

$$
(\omega \theta)_{x}=-\omega^{2}\left(\frac{\varphi}{\omega}\right)_{y}, \quad(\omega \theta)_{y}=\omega^{2}\left(\frac{\varphi}{\omega}\right)_{x}
$$

удовлетворяет уравнению $\widetilde{H} \theta=0$.

Очевидно, что если $\theta$ удовлетворяет (3), то

$$
\theta+\frac{C}{\omega}, \quad C=\text { const }
$$

удовлетворяет (3) для любой постоянной $C$.

Мы будем использовать следующее обозначение для преобразования Мутара:

$$
M_{\omega}(u)=\tilde{u}=u-2 \Delta \ln \omega, \quad M_{\omega}(\varphi)=\left\{\theta+\frac{C}{\omega}, C \in \mathbb{C}\right\} .
$$

В одномерном пределе преобразование Мутара сводится к преобразованию Дарбу. Действительно, пусть $u=u(x)$ зависит лишь от $x$, а $\omega=f(x) e^{\sqrt{c} y}$. В этом случае функция $f$ удовлетворяет одномерному уравнению Шредингера

$$
H_{0} f=\left(-\frac{d^{2}}{d x^{2}}+u\right) f=c f
$$


и преобразование Мутара сводится к преобразованию Дарбу оператора $H_{0}$, определенному решением $f$ :

$$
H=H_{0}-\frac{\partial^{2}}{\partial y^{2}} \rightarrow \widetilde{H}=\widetilde{H}_{0}-\frac{\partial^{2}}{\partial y^{2}}
$$

Если $g=g(x)$ удовлетворяет уравнению $H_{0} g=E g$, то $H \varphi=0$, где $\varphi=e^{\sqrt{E} y} g(x)$. Из (3) заключаем, что $\theta=e^{\sqrt{E}} y(x)$ удовлетворяет уравнению $\widetilde{H} \theta=0$, если

$$
h=\frac{1}{\sqrt{c}+\sqrt{E}}\left(\frac{d}{d x}-\frac{f_{x}}{f}\right) g,
$$

т.е. $h$ отличается от преобразования Дарбу функции $g$ лишь множителем:

$$
h=-\frac{1}{\sqrt{c}+\sqrt{E}} A g .
$$

Здесь $H_{0}-c=A^{\mathrm{T}} A-$ факторизация оператора $H_{0}-c$, определенная $f$. Обратное преобразование Дарбу задается формулой

$$
g=\frac{1}{\sqrt{c}-\sqrt{E}}\left(\frac{d}{d x}+\frac{f_{x}}{f}\right) h .
$$

ЗАмечАние 2. Существует другое двумерное обобщение преобразования Дарбу - каскадное преобразование Лапласа. Оно определено для более общих операторов, т.е. операторов Шредингера с электромагнитным полем

$$
H=4(\bar{\partial}+\beta)(-\partial+\alpha)+u,
$$

и задается формулой

$$
H \rightarrow \widetilde{H}=4 u(-\partial+\alpha) u^{-1}(\bar{\partial}+\beta)+u
$$

Тем самым, если $H \varphi=0$, то $\widetilde{\varphi}=(-\partial+\alpha) \varphi$ удовлетворяет уравнению $\widetilde{H} \widetilde{\varphi}=0$. В одномерном пределе указанное преобразование также сводится к преобразованию Дарбу. Его связь с интегрируемыми системами была недавно изучена в [13].

\section{3. СОЛИТОННЫЕ ПОТЕНЦИАЛЫ, ПОЛУЧАЕМЫЕ С ПОМОЩЬЮ ПРЕОБРАЗОВАНИЯ МУТАРА}

В [2] мы привели простейшие примеры быстроубывающих рациональных потенциалов двумерного оператора Шредингера, имеющих многомерное $L_{2}$-ядро. Эти примеры были построены с помощью преобразования Мутара следующим образом.

ОсНОВНАЯ КОНСТРУКция. Пусть

$$
H_{0}=-\Delta=-\Delta+u_{0}
$$

- оператор с потенциалом $u_{0}=0, \omega_{1}$ и $\omega_{2}$ удовлетворяют соответственно уравнениям

$$
H_{0} \omega_{1}=0, \quad H_{0} \omega_{2}=0 .
$$

2 Теоретическая и математическая физика, т. 157, № 2, 2008 г. 
Применим преобразования Мутара $M_{\omega_{1}}$ и $M_{\omega_{2}}$, определенные решениями $\omega_{1}, \omega_{2}$, и получим операторы

$$
H_{1}=-\Delta+u_{1}, \quad H_{2}=-\Delta+u_{2},
$$

где $u_{1}=M_{\omega_{1}}\left(u_{0}\right), u_{2}=M_{\omega_{2}}\left(u_{0}\right)$. По построению имеем

$$
H_{1} M_{\omega_{1}}\left(\omega_{2}\right)=0, \quad H_{2} M_{\omega_{2}}\left(\omega_{1}\right)=0 .
$$

Выберем некоторую функцию $\theta_{1} \in M_{\omega_{1}}\left(\omega_{2}\right)$ и положим

$$
\theta_{2}=-\frac{\omega_{1}}{\omega_{2}} \theta_{1} \in M_{\omega_{2}}\left(\omega_{1}\right)
$$

Эти функции определяют преобразования Мутара операторов $H_{1}$ и $H_{2}$. В результате получаем операторы $H_{12}$ и $H_{21}$ с потенциалами

$$
u_{12}=M_{\theta_{1}}\left(u_{1}\right), \quad u_{21}=M_{\theta_{2}}\left(u_{2}\right) .
$$

Следующая лемма может быть доказана прямым вычислением, которое мы опускаем.

Лемма. 1) $u_{12}=u_{21}=u$, m.e. диаграмма

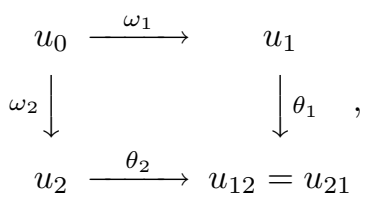

где $\theta_{1} \in M_{\omega_{1}}\left(\omega_{2}\right), \theta_{2}=-\omega_{1} \theta_{1} / \omega_{2} \in M_{\omega_{2}}\left(\omega_{1}\right)$, коммутативна;

2) для $\psi_{1}=1 / \theta_{1}$ и $\psi_{2}=1 / \theta_{2}$ имеем

$$
H \psi_{1}=H \psi_{2}=0
$$

где $H=-\Delta+u$.

Отметим, что в приведенной конструкции имеется свободный скалярный параметр $C(4)$, соответствующий выбору конкретной функции $\theta_{1} \in M_{\omega_{1}}\left(\omega_{2}\right)$. Этот параметр в некоторых случаях может быть использован для построения несингулярного потенциала $u$ и функций $\psi_{1}$ и $\psi_{2}$.

Применим теперь описанную конструкцию для случая $u_{0}=0$. Искомая явная формула для нахождения потенциала $u$ приводится в следующей теореме 1 . Она также может быть доказана прямым вычислением, которое мы опускаем.

TeOpema 1. Пyсmь

$$
\omega_{1}=p_{1}(z)+\overline{p_{1}(z)}, \quad \omega_{2}=p_{2}(z)+\overline{p_{2}(z)},
$$


где $p_{1}, p_{2}$ - голоморфные функиии от z. Рассмотрим преобразование Мутара оператора $H_{0}=-\Delta$, определенное $\omega_{1}$. Тогда соответствующее преобразование (3) от $\omega_{2}$ задается формулой

$$
\theta_{1}=\frac{i}{p_{1}+\bar{p}_{1}}\left(\left(p_{1} \bar{p}_{2}-p_{2} \bar{p}_{1}\right)+\int\left(\left(p_{1}^{\prime} p_{2}-p_{1} p_{2}^{\prime}\right) d z+\left(\bar{p}_{1} \bar{p}_{2}^{\prime}-\bar{p}_{1}^{\prime} \bar{p}_{2}\right) d \bar{z}\right)\right) .
$$

Тем самым вторая итерация, определенная бункиией $\theta_{1}$, дает нам оператор $H=$ $-\Delta+u$, где

$$
\begin{aligned}
u & =-2 \Delta \ln \omega_{1}-2 \Delta \ln \theta_{1}=-2 \Delta \ln \left(\omega_{1} \theta_{1}\right)= \\
& =-2 \Delta \ln i\left(\left(p_{1} \bar{p}_{2}-p_{2} \bar{p}_{1}\right)+\int\left(\left(p_{1}^{\prime} p_{2}-p_{1} p_{2}^{\prime}\right) d z+\left(\bar{p}_{1} \bar{p}_{2}^{\prime}-\bar{p}_{1}^{\prime} \bar{p}_{2}\right) d \bar{z}\right)\right) .
\end{aligned}
$$

Свободный скалярный параметр, который мы упомянули ранее, появляется здесь как постоянная интегрирования в (5).

Применим теорему 1 для получения некоторых интересных примеров операторов Шредингера. Ниже мы будем рассматривать лишь случаи, в которых $\omega_{1}$ и $\omega_{2}-$ вещественнозначные гармонические многочлены.

ПРИМЕР 1. Пусть

$$
\omega_{1}=x+2\left(x^{2}-y^{2}\right)+x y, \quad \omega_{2}=x+y+\frac{3}{2}\left(x^{2}-y^{2}\right)+5 x y .
$$

Функции $\omega_{1}, \omega_{2}$ в терминах полиномов от комплексной переменной $z$ можно записать в виде

$$
p_{1}(z)=\left(1-\frac{i}{4}\right) z^{2}+\frac{z}{2}, \quad p_{2}(z)=\frac{1}{4}(3-5 i) z^{2}+\frac{1-i}{2} z .
$$

При некотором подходящем значении постоянной $C$ в $\theta_{1}$ получаем

$$
\begin{aligned}
u=- & \frac{5120\left(1+8 x+2 y+17 x^{2}+17 y^{2}\right)}{\left(160+4 x^{2}+4 y^{2}+16 x^{3}+4 x^{2} y+16 x y^{2}+4 y^{3}+17\left(x^{2}+y^{2}\right)^{2}\right)^{2}}= \\
=- & \frac{5120|1+(4-i) z|^{2}}{\left(160+|z|^{2}|2+(4-i) z|^{2}\right)^{2}}, \\
& \psi_{1}=\frac{x+2 x^{2}+x y-2 y^{2}}{160+4 x^{2}+4 y^{2}+16 x^{3}+4 x^{2} y+16 x y^{2}+4 y^{3}+17\left(x^{2}+y^{2}\right)^{2}}, \\
& \psi_{2}=\frac{2 x+2 y+3 x^{2}+10 x y-3 y^{2}}{160+4 x^{2}+4 y^{2}+16 x^{3}+4 x^{2} y+16 x y^{2}+4 y^{3}+17\left(x^{2}+y^{2}\right)^{2}}
\end{aligned}
$$

В (8) мы упростили полученные выражения для $\psi_{1}$ и $\psi_{2}$, умножив их на подходящие постоянные. На рис. 1-3 мы приводим графики потенциала $u$ и решений $\psi_{1}, \psi_{2}$.

ТЕОРема 2 [2]. Потенииал и, заданный формулой (7), является гладким раицональным и убъвает как $1 / r^{6}$ при $r \rightarrow \infty$. Функции $\psi_{1}$ u $\psi_{2}$, заданнве в (8), являются гладкими рациональными, убывают как $1 / r^{2}$ при $r \rightarrow \infty$ и порождают двумерное подпространство в ядре оператора $L=-\Delta+u: L_{2}\left(\mathbb{R}^{2}\right) \rightarrow L_{2}\left(\mathbb{R}^{2}\right)$. 


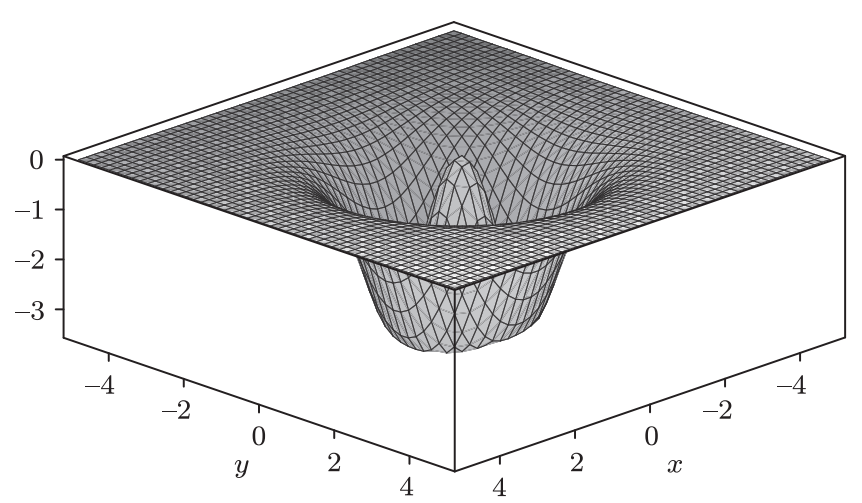

Рис. 1. Потенциал (7)

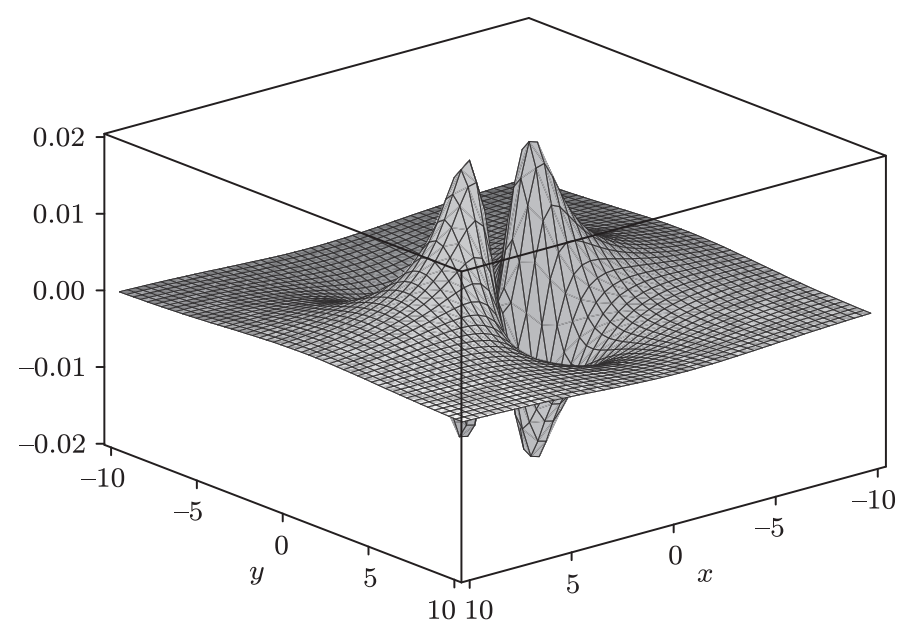

Рис. 2. Решение $\psi_{1}$ в (8).

Здесь и в следующем примере мы полагаем $r=\sqrt{x^{2}+y^{2}}$.

Пример 2. Возьмем в качестве функций $\omega_{1}$ и $\omega_{2}$ два гармонических полинома третьей степени:

$$
\begin{aligned}
& \omega_{1}=x+\frac{x^{2}-y^{2}-3 x y}{5}+2\left(-x^{3}-3 x^{2} y+3 x y^{2}+y^{3}\right) \\
& \omega_{2}=x+y+\frac{x^{2}-y^{2}}{2}-\frac{x y}{5}-4\left(3 x^{2} y-y^{3}\right)
\end{aligned}
$$

которые в терминах полиномов от комплексной переменной $z$ имеют вид

$$
p_{1}(z)=(i-1) z^{3}+\left(\frac{1}{10}+\frac{3}{20} i\right) z^{2}+\frac{z}{2}, \quad p_{2}(z)=2 i z^{3}+\left(\frac{1}{4}+\frac{i}{20}\right) z^{2}+\frac{1-i}{2} z
$$




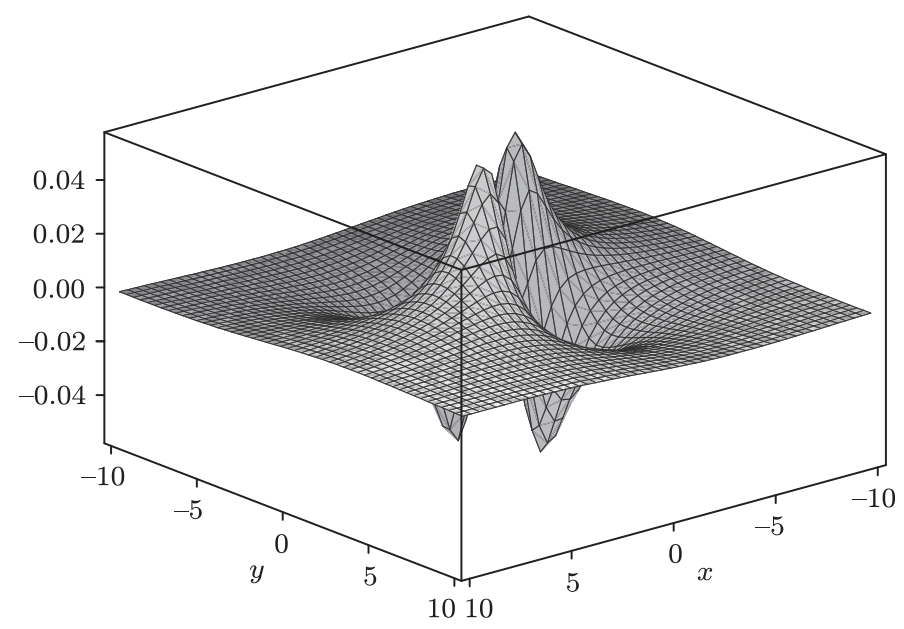

Рис. 3. Решение $\psi_{2}$ в (8).

Тогда потенциал $u$ и функции $\psi_{1}$ и $\psi_{2}$ принимают вид

$$
u=\frac{F_{0}(x, y)}{G(x, y)^{2}}, \quad \psi_{1}=\frac{F_{1}(x, y)}{G(x, y)}, \quad \psi_{2}=\frac{F_{2}(x, y)}{G(x, y)},
$$

где

$$
\begin{aligned}
& F_{0}(x, y)=-1280000\left(25+20 x-287 x^{2}+60 x^{3}+1800 x^{4}-30 y-600 x y-\right. \\
& \left.-300 x^{2} y+313 y^{2}+60 x y^{2}+3600 x^{2} y^{2}-300 y^{3}+1800 y^{4}\right), \\
& G(x, y)=40000+100 x^{2}+40 x^{3}-387 x^{4}+40 x^{5}+800 x^{6}-60 x^{2} y- \\
& -800 x^{3} y-200 x^{4} y+100 y^{2}+40 x y^{2}+26 x^{2} y^{2}+80 x^{3} y^{2}+2400 x^{4} y^{2}- \\
& -60 y^{3}-800 x y^{3}-400 x^{2} y^{3}+413 y^{4}+40 x y^{4}+2400 x^{2} y^{4}-200 y^{5}+800 y^{6} \text {, } \\
& F_{1}(x, y)=-10 x-2 x^{2}+20 x^{3}+6 x y+60 x^{2} y+2 y^{2}-60 x y^{2}-20 y^{3} \text {, } \\
& F_{2}(x, y)=-10 x-5 x^{2}-10 y+2 x y+120 x^{2} y+5 y^{2}-40 y^{3}
\end{aligned}
$$

(как и в предыдущем примере, мы упростили выражения для $\psi_{1}$ и $\psi_{2}$, умножив их на подходящие постоянные). На рис. 4-6 мы приводим графики потенциала $u$ и решений $\psi_{1}, \psi_{2}$.

ТЕОРема 3 [2]. Потенциал и, заданный в (10), является гладким рациональным и убывает как $1 / r^{8}$ при $r \rightarrow \infty$. Функиии $\psi_{1} u \psi_{2}$, заданные формулами (10), являются гладкими рациональными, убывают как $1 / r^{3}$ при $r \rightarrow \infty$ и порождают двумерное подпространство в ядре оператора $L=-\Delta+u: L_{2}\left(\mathbb{R}^{2}\right) \rightarrow L_{2}\left(\mathbb{R}^{2}\right)$.

ЗАмЕчАниЕ 3. Естественно предположить, что для любого $N>0$, применяя описанную выше конструкцию к другим гармоническим многочленам, можно построить гладкие рациональные потенциалы $u$ и собственные функции $\psi_{1}$ и $\psi_{2}$, убывающие быстрее $1 / r^{N}$. 


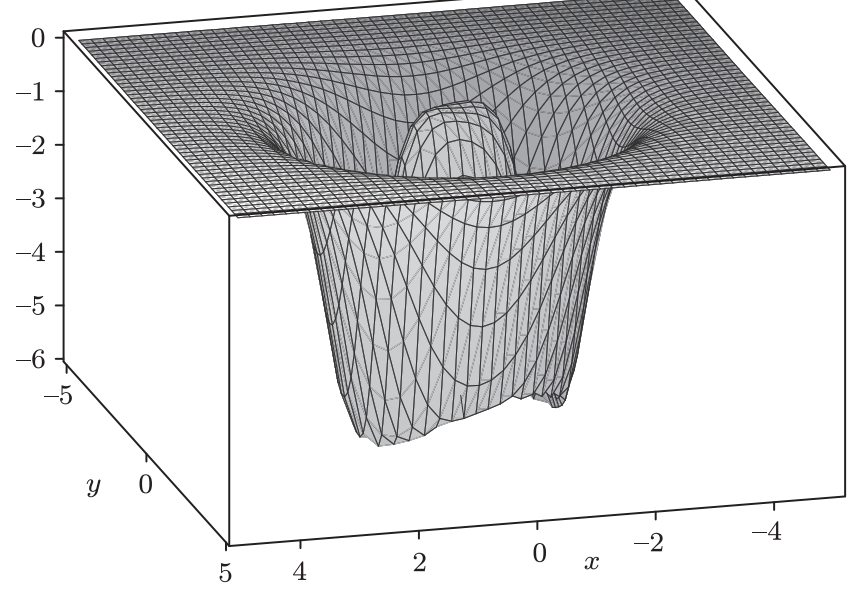

Рис. 4. Потенциал (10).

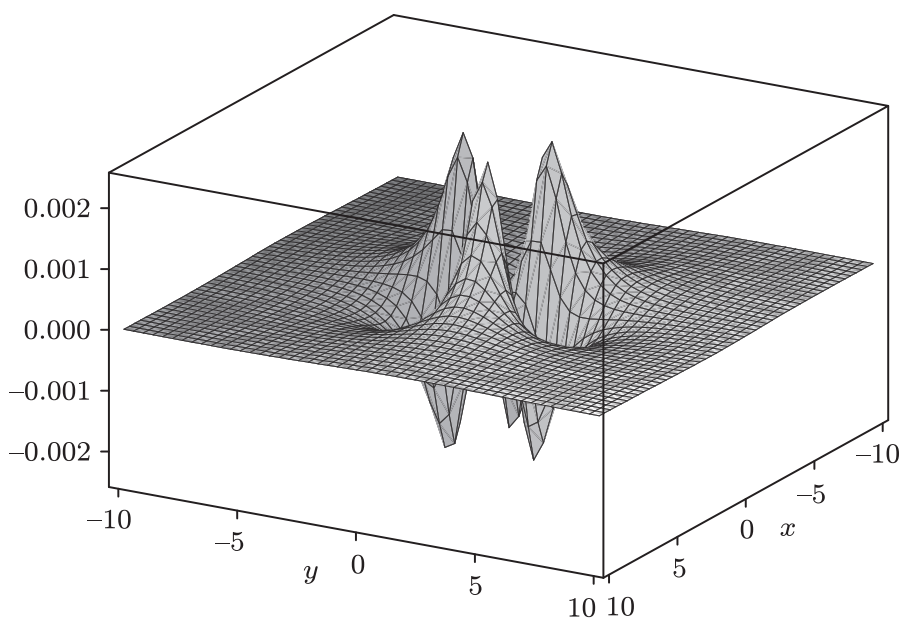

Рис. 5. Решение $\psi_{1}$ в (10).

\section{4. СОЛИТОННЫЕ УРАВНЕНИЯ}

4.1. Явные решения уравнения ВН. Для простоты в данном разделе мы перенормируем оператор Шредингера

$$
H=\partial \bar{\partial}+U=\frac{1}{4} \Delta-\frac{u}{4}
$$

используя стандартные операторы $\partial=\partial_{z}=\left(\partial_{x}-i \partial_{y}\right) / 2, \bar{\partial}=\partial_{\bar{z}}=\left(\partial_{x}+i \partial_{y}\right) / 2$. Преобразование Мутара, определяемое функцией $\omega$, принимает вид

$$
U \rightarrow U+2 \partial \bar{\partial} \ln \omega
$$




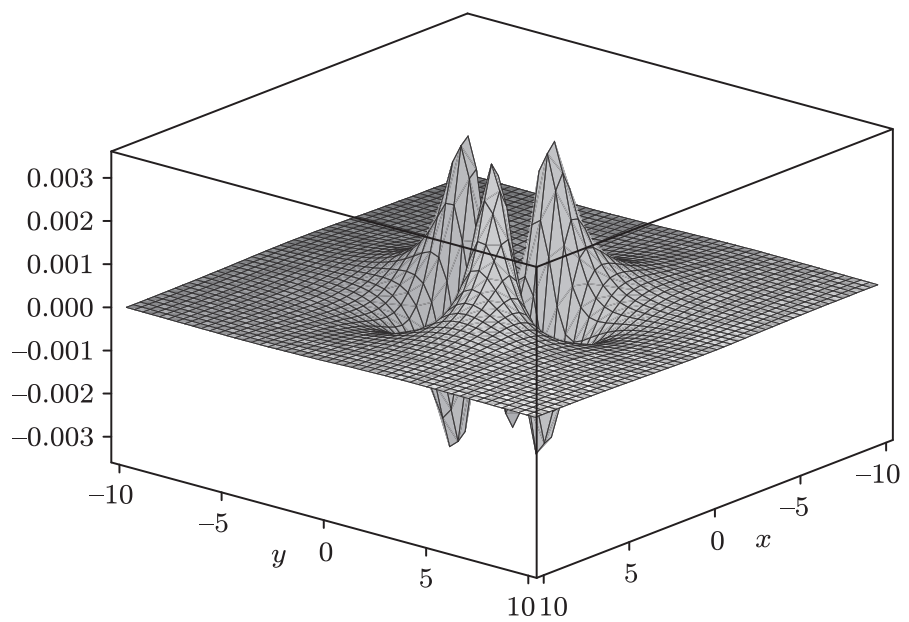

Рис. 6. Решение $\psi_{2}$ в (10).

а преобразование собственных функций, которое задается теми же формулами (3), следует переписать в терминах комплексной переменной в виде

$$
\left(\bar{\partial}+\frac{\omega_{\bar{z}}}{\omega}\right) \theta=i\left(\bar{\partial}-\frac{\omega_{\bar{z}}}{\omega}\right) \varphi, \quad\left(\partial+\frac{\omega_{z}}{\omega}\right) \theta=-i\left(\partial-\frac{\omega_{z}}{\omega}\right) \varphi
$$

или

$$
\varphi \rightarrow \theta=\frac{i}{\omega} \int(\varphi \partial \omega-\omega \partial \varphi) d z-(\varphi \bar{\partial} \omega-\omega \bar{\partial} \varphi) d \bar{z} .
$$

Будем предполагать, что функция $\varphi$ зависит также от времени $t$ и удовлетворяет уравнениям

$$
H \varphi=0, \quad \partial_{t} \varphi=\left(\partial^{3}+\bar{\partial}^{3}+3 V \partial+3 V^{*} \partial\right) \varphi,
$$

где $\bar{\partial} V=\partial U, \partial V^{*}=\bar{\partial} U$. Непосредственным вычислением доказывается

УтвеРЖДЕНИЕ 3 (Расширенное преобразование Мутара) ${ }^{1)}$. Cистема (12) инвариантна относительно расширенного преобразования Мутара

$$
\begin{aligned}
\varphi \rightarrow \theta=\frac{i}{\omega} & \int(\varphi \partial \omega-\omega \partial \varphi) d z-(\varphi \bar{\partial} \omega-\omega \bar{\partial} \varphi) d \bar{z}+ \\
& +\left[\varphi \partial^{3} \omega-\omega \partial^{3} \varphi+\omega \bar{\partial}^{3} \varphi-\varphi \bar{\partial}^{3} \omega+2\left(\partial^{2} \varphi \partial \omega-\partial \varphi \partial^{2} \omega\right)-\right. \\
& \left.-2\left(\bar{\partial}^{2} \varphi \bar{\partial} \omega-\bar{\partial} \varphi \bar{\partial}^{2} \omega\right)+3 V(\varphi \partial \omega-\omega \partial \varphi)+3 V^{*}(\omega \bar{\partial} \varphi-\varphi \bar{\partial} \omega)\right] d t \\
U \rightarrow U & +2 \partial \bar{\partial} \ln \omega, \quad V \rightarrow V+2 \partial^{2} \ln \omega, \quad V^{*} \rightarrow V^{*}+2 \bar{\partial}^{2} \ln \omega .
\end{aligned}
$$

Если $\omega$ - вещественнозначная функиия, то указанные преобразования сохраняют свойство $V^{*}=\bar{V}$.

1)Эта теорема впервые была сформулирована в [14]. Однако в [14] третья строка в формуле преобразования Мутара $\varphi \rightarrow \theta$ была пропущена. В настоящей работе мы исправляем эту ошибку. Вследствие указанной ошибки формула (6.2.5) в работе [14] не дает решения уравнения ВН. Недавно мы узнали, что это исправление уже было сделано в [15] (см. также [16], где был описан другой подход к расширенным преобразованиям Мутара). 
Условием совместности системы (12) является система

$$
\begin{gathered}
U_{t}=\partial^{3} U+\bar{\partial}^{3} U+3 \partial(U V)+3 \bar{\partial}\left(V^{*} U\right)=0, \\
\bar{\partial} V=\partial U, \quad \partial V^{*}=\bar{\partial} U
\end{gathered}
$$

которая при $V^{*}=\bar{V}$ сводится к уравнению ВН. Ниже мы будем рассматривать только этот случай.

Рассмотрим следующий поток на пространстве функций $p(z, t)$, голоморфных по $z$ :

$$
\frac{\partial p}{\partial t}=\frac{\partial^{3} p}{\partial z}
$$

Если функции $p_{1}(z, t)$ и $p_{2}(z, t)$ удовлетворяют $(14)$, то функции $\omega=p_{1}+\bar{p}_{1}$ и $\varphi=p_{2}+\bar{p}_{2}$ удовлетворяют системе (12) при $U=V=0$. Расширенное преобразование Мутара отличается от классического на слагаемое с дифференциалом $d t$ :

$$
\theta=\frac{i}{\omega} \int\left(\Psi_{1}(\omega, \varphi) d z+\Psi_{2}(\omega, \varphi) d \bar{z}+\Theta(\omega, \varphi) d t\right) .
$$

Подставим $\omega=p_{1}+\bar{p}_{1}, \varphi=p_{2}+\bar{p}_{2}$ в $\Psi_{s}$. В соответствии с (14) и равенствами $V=V^{*}=0$ подынтегральное выражение является замкнутой формой, что влечет равенства

$$
\begin{aligned}
& \frac{\partial \Psi_{1}\left(p_{1}+\bar{p}_{1}, p_{2}+\bar{p}_{2}\right)}{\partial t}=\frac{\partial \Theta\left(p_{1}+\bar{p}_{1}, p_{2}+\bar{p}_{2}\right)}{\partial z} \\
& \frac{\partial \Psi_{2}\left(p_{1}+\bar{p}_{1}, p_{2}+\bar{p}_{2}\right)}{\partial t}=\frac{\partial \Theta\left(p_{1}+\bar{p}_{1}, p_{2}+\bar{p}_{2}\right)}{\partial \bar{z}} .
\end{aligned}
$$

Следовательно, функции $\Theta_{z}$ и $\Theta_{\bar{z}}$ определяются по $\Psi_{s}$ и мы имеем следующий аналог теоремы 1.

УТВЕРЖДЕНИЕ 4. Пусть

$$
\omega_{1}=p_{1}(z, t)+\overline{p_{1}(z, t)}, \quad \omega_{2}=p_{2}(z, t)+\overline{p_{2}(z, t)}
$$

где $p_{1}$ и $p_{2}$ - голоморфные функиии от $z$, удовлетворяющие (14). Рассмотрим расширенное преобразование Мутара оператора $H_{0}=-\Delta$, определенное $\omega_{1}$. Пусть $\theta_{1}$ - образ $\omega_{2}$ под действием этого преобразования и пусть $H=-\Delta+u$ получен итерацией преобразования Мутара, определенного $\theta_{1}$. Тогда

$$
\begin{aligned}
& U(z, \bar{z}, t)=2 \partial \bar{\partial} \ln i\left(\left(p_{1} \overline{p_{2}}-p_{2} \overline{p_{1}}\right)+\int\left(\left(p_{1}^{\prime} p_{2}-p_{1} p_{2}^{\prime}\right) d z+\left({\overline{p_{1}}}_{{\overline{p_{2}}}^{\prime}}-{\overline{p_{1}}}^{\prime} \overline{p_{2}}\right) d \bar{z}\right)+\right. \\
& \left.+\int\left(p_{1}^{\prime \prime \prime} p_{2}-p_{1} p_{2}^{\prime \prime \prime}+2\left(p_{1}^{\prime} p_{2}^{\prime \prime}-p_{1}^{\prime \prime} p_{2}^{\prime}\right)+{\overline{p_{1}}}_{\bar{p}_{2}}{ }^{\prime \prime \prime}-{\overline{p_{1}}}^{\prime \prime \prime} \overline{p_{2}}+2\left({\overline{p_{1}}}^{\prime \prime}{\overline{p_{2}}}^{\prime}-{\overline{p_{1}}}^{\prime}{\overline{p_{2}}}^{\prime \prime}\right)\right) d t\right) \text {. }
\end{aligned}
$$

СлеДСТВИЕ 1. Пусть $p_{1}(z, t)$ u $p_{2}(z, t)$ - голоморфные функиии от $z$, которье удовлетворяют уравнению (14). Тогда подстановка их в (15) дает решение уравнения ВН, рачиональное по z, $\bar{z} u t$.

ЗАмЕчАниЕ 4. Как очевидно, аналоги (13) существуют для всех уравнений иерархии ВН, и, следовательно, могут быть получены явные формулы для их решений, в частности, вида (13). 
4.2. $\sigma$-Потоки. Обозначим через $\mathcal{H}_{N}$ пространство всех гармонических многочленов $p(z)+\overline{p(z)}$, где

$$
p(z)=\sigma_{0} z^{N}+\sigma_{1} z^{N-1}+\cdots+\sigma_{N-1} z+\sigma_{N},
$$

и через $\mathcal{H}_{N}^{0}-$ подпространство при $\sigma_{0}=1$.

Поток (14) порождает на $\mathcal{H}_{N}$ линейный поток

$$
\dot{\sigma}_{k}=(k-1)(k-2)(k-3) \sigma_{k+3}, \quad k=0,1, \ldots, N .
$$

Поскольку $\sigma_{0}$ постоянна вдоль потока, мы можем ограничить эти уравнения на $\mathcal{H}_{N}^{0}$ и получить следующий частный вид линейной системы на $\sigma_{1}, \ldots, \sigma_{N}$ :

$$
\dot{\sigma}=A+B \sigma,
$$

которая порождает динамическую систему на $n$-й симметрической степени $S^{n} \mathbb{C}$ пространства $\mathbb{C}$. Действительно, $\sigma_{1}, \ldots, \sigma_{n}$ являются элементарными симметрическими многочленами от корней $z_{1}, \ldots, z_{n}$ многочлена $p(z)$ :

$$
\sigma_{1}\left(z_{1}, \ldots, z_{n}\right)=-\left(z_{1}+\cdots+z_{n}\right), \quad \ldots, \quad \sigma_{n}\left(z_{1}, \ldots, z_{n}\right)=(-1)^{n} z_{1} \ldots z_{n},
$$

и интегрируемая (фактически линейная) эволюция $\sigma$ порождает на $S^{n} \mathbb{C}$ некоторую динамическую систему. Мы будем называть эту динамическую систему на $S^{n} \mathbb{C}$ $\sigma$-системой.

В отличие от системы Калоджеро-Мозера, $\sigma$-системы не описывают динамику сингулярностей уравнения ВН (система Калоджеро-Мозера дает эту динамику для уравнения КдФ), т.е. динамику частицеподобных решений [17], [12] (см. также п. 2.2 настоящей работы). Фактически системы Калоджеро-Мозера и $\sigma$-системы различны во многих отношениях.

K примеру, рассмотрим простейший поток, порожденный (14) на $\mathcal{H}_{3}$ :

$$
\dot{\sigma}_{1}=0, \quad \dot{\sigma}_{2}=0, \quad \dot{\sigma}_{3}=6 \text {. }
$$

Общее решение дается формулами

$$
\sigma_{1}=a_{1}, \quad \sigma_{2}=a_{2}, \quad \sigma_{3}=a_{3}+6 t .
$$

Однако для решений $\left(x_{1}(t), \ldots, x_{n}(t)\right)$ системы Калоджеро-Мозера $k$-й элементарный симметрический многочлен $\sigma_{k}\left(x_{1}, \ldots, x_{n}\right)$ является полиномом по $t$ степени $k$, и, следовательно, любая функция $x_{k}(t)$ является алгебраической ([12], следствие 3$)$.

Мы можем привести примеры, в которых решения системы Калоджеро-Мозера и $\sigma$-системы связаны перепараметризацией временно́й переменной. Таким решением, например, является решение "равностороннего треугольника" системы КалоджероМозера, которое соответствует решению

$$
-2 \frac{d^{2}}{d x^{2}}\left[\left(x-x_{1}(t)\right)\left(x-x_{2}(t)\right)\left(x-x_{3}(t)\right)\right]
$$


уравнения КдФ:

$$
x_{k}(t)=\varepsilon^{k} \sqrt[3]{\varepsilon} t, \quad \varepsilon=e^{2 \pi i / 3}, \quad k=1,2,3,
$$

и решение $\sigma$-системы, соответствующее $p(z, t)=z^{3}+6 t$ :

$$
z_{k}(t)=\varepsilon^{k} \sqrt[3]{6 t}, \quad \varepsilon=e^{2 \pi i / 3}, \quad k=1,2,3 .
$$

Согласно следствию 1 имеем следующее утверждение.

Если даны два решения $p_{1} \in \mathcal{H}_{M}^{0}$ и $p_{2} \in \mathcal{H}_{N}^{0}$ системы (16), то подстановкой $e^{i \lambda_{1}} p_{1}$ и $e^{i \lambda_{2}} p_{2}$ (где $\lambda_{1}$ и $\lambda_{2}$ - вещественные постоянные) в (15) получим решения уравнения $\mathrm{BH}^{2)}$ Тем самым каждой паре решений (16) соответствует $\left(S^{1} \times S^{1}\right)$-семейство решений уравнения $\mathrm{BH}$.

Мы полагаем, что $\sigma$-системы заслуживают отдельного изучения не только в силу установленной нами связи с уравнением ВН, но и в силу естественного характера их появления в данном круге задач.

4.3. О распаде решений. Для простоты запишем формулу (15) в виде

$$
U=2 \partial \bar{\partial} \ln \Phi\left(p_{1}, p_{2}\right)
$$

(см. формулу (11) новой нормировки операторов). В результате интегрирования в $(5)$ функция $\Phi\left(p_{1}, p_{2}\right)$ определена с точностью до постоянной, которую мы предполагаем вещественной, что приводит к вещественному потенциалу $U(z, \bar{z})$. Согласно утверждению 3 потенциал $V=\bar{V}^{*}$ равен

$$
V=2 \partial^{2} \ln \Phi\left(p_{1}, p_{2}\right) \text {. }
$$

Легко проверить справедливость следующего утверждения.

СлЕДСТвиЕ 2. Потенииал (7) (см. теорему 2) является стационарным решением уравнения $\mathrm{BH}$.

С другой стороны, с помощью предложенного выше метода можно получить решения уравнения ВН, имеющие сингулярности при $t>0$.

Теорема 4. Решение $U(z, \bar{z}, t)$ уравнения $B H$, полученное из полиномов $p_{1}=i z^{2}$, $p_{2}=z^{2}+(1+i) z$ в соответствии с утверждением 4 при условии, что постоянная интегрирования в (13) $C=-20$, имеет вид

$$
U=\frac{H_{1}}{H_{2}}
$$

əəe

$$
\begin{aligned}
H_{1}=- & 12\left(24 t x^{2}+12 t x+24 t y^{2}+12 t y+x^{5}-3 x^{4} y+2 x^{4}-2 x^{3} y^{2}-4 x^{3} y-2 x^{2} y^{3}-\right. \\
& \left.-60 x^{2}-3 x y^{4}-4 x y^{3}-30 x+y^{5}+2 y^{4}-60 y^{2}-30 y\right),
\end{aligned}
$$

${ }^{2)}$ Если умножить, к примеру, $p_{1}$ на постоянную $\mu \in \mathbb{R}$, то $\omega=p_{1}+\bar{p}_{1}$ умножится на $\mu$, и, поскольку преобразование Мутара зависит от выбора $\omega$, взятого с точностью до постоянного множителя, это не изменит результата преобразования. 


$$
H_{2}=\left(3 x^{4}+4 x^{3}+6 x^{2} y^{2}+3 y^{4}+4 y^{3}+30-12 t\right)^{2} \text {. }
$$

Это решение убъвает на бесконечности как $r^{-3}$ и, очевидно, имеет сингулярность при достаточно больиих значениях $t>0$.

\section{5. КУБИЧЕСКАЯ ФОРМУЛА СУПЕРПОЗИЦИИ}

В данном разделе мы распространим алгебраическую формулу суперпозиции Бьянки [18] для трех начальных решений $\omega_{1}, \omega_{2}, \omega_{3}$ уравнения Мутара на случай уравнения ВН. Фактически уже расширенное преобразование Мутара (13) разумно рассматривать как "расширенную формулу суперпозиции", которая строит новое решение $\theta$ по двум начальным решениям $\omega, \varphi$ линейной задачи (12) с некоторыми начальными потенциалами $U, V, V^{*}$. В отличие от широко известной алгебраической формулы суперпозиции двух решений уравнения синус-Гордон, преобразование (13) не является алгебраической операцией и требует выполнения квадратуры. Это типично для $(2+1)$-мерных преобразований Беклунда. В этом случае алгебраическая формула суперпозиции существует для трех начальных решений (см. [19], где дано более детальное изложение свойств многомерных преобразований Беклунда).

Легко проверить, что следующее предложение, полученное Бьянки [18] для уравнения Мутара $\varphi_{x y}=u(x, y) \varphi$, также верно и в нашем случае.

Теорема 5. Пусть $\omega_{1}, \omega_{2}, \omega_{3}$ - три решения (12), где $U=U_{0}, V=V_{0}, V^{*}=$ $V_{0}^{*}$ - некоторое заданное решение уравнения $B H(1), \theta_{1}, \theta_{2}$ - преобразованные решения (12) с новыми потенциалами $U_{1}=U_{0}-2 \partial \bar{\partial}\left(\ln \omega_{1}\right), U_{2}=U_{0}-2 \partial \bar{\partial}\left(\ln \omega_{2}\right)$ u соответственно преобразованными $V_{i}, V_{i}^{*}$ (другим решением уравнения ВН), полученные из $\omega_{3}$ с помощъю (13). Тогда существует единственное решение $\theta^{\prime}$ четвертой линейной системы (12) с потенциалами $U=U_{12}, V=V_{12}, V^{*}=V_{12}^{*}$ такое, что $\theta^{\prime}$ связано с $\theta_{1}, \theta_{2}$ посредством расширенного преобразования Мутара (13). Это решение $\theta^{\prime}$ выражается алгебраической формулой

$$
\theta^{\prime}-\omega_{3}=\frac{\omega_{1} \omega_{2}}{\lambda}\left(\theta_{2}-\theta_{1}\right), \quad \lambda=\omega_{1} \omega_{1}^{\prime}=-\omega_{2} \omega_{2}^{\prime},
$$

где $\omega_{1}^{\prime}, \omega_{2}^{\prime}$ полученъц из $\omega_{1}, \omega_{2}$ в соответствии с (13).

Представим полученное утверждение и "расширенную формулу суперпозиции" (13) в виде коммутативных ромбической и кубической диаграмм ("куб Бьянки нелинейной суперпозиции"), показанных на рис. 7.

Ромбическая диаграмма на рис. 7а показывает решения $\omega_{1}$ и $\omega_{2}$ системы (12) с начальным потенциалом $U_{0}$ и соответствующими потенциалами $V_{0}, V_{0}^{*}$, которые дают некоторое решение уравнения ВН (1). Эти решения $\omega_{i}$ определяют два новых потенциала $U_{1}, U_{2}$, как и соответствующие потенциалы $V_{i}, V_{i}^{*}$, два новых решения уравнения ВН. Это является первъм шагом нелинейной суперпозиции. Согласно формуле (13) имеется однопараметрическое множество решений $\theta_{1}$ системы (12) с потенциалами $U_{1}, V_{1}, V_{1}^{*}$, а также $\theta_{2}=-\omega_{1} \theta_{1} / \omega_{2}$ - решение (12) с потенциалами $U_{2}$, 


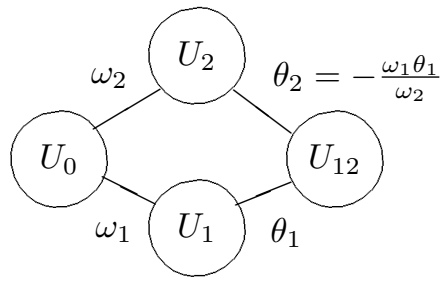

$a$

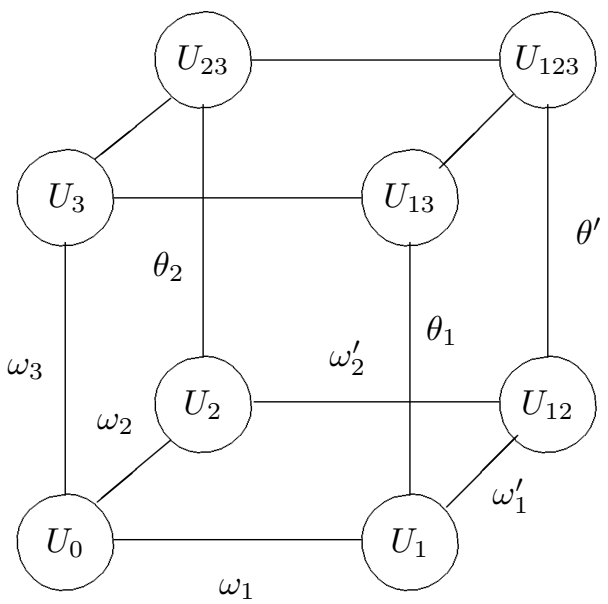

6

Рис. 7. Ромбическая и кубическая формулы нелинейной суперпозиции для $(2+1)$-мерных интегрируемых систем.

$V_{2}, V_{2}^{*}$. Используя далее либо $\theta_{1}$, либо $\theta_{2}$, мы можем найти потенциалы

$$
\begin{aligned}
& U_{12}=U_{1}+2 \partial \bar{\partial} \ln \theta_{1}=U_{2}+2 \partial \bar{\partial} \ln \theta_{2}, \\
& V_{12}=V_{1}+2 \partial^{2} \ln \theta_{1}=V_{2}+2 \partial^{2} \ln \theta_{2}, \\
& V_{12}^{*}=V_{1}^{*}+2 \bar{\partial}^{2} \ln \theta_{1}=V_{2}^{*}+2 \bar{\partial}^{2} \ln \theta_{2},
\end{aligned}
$$

показанные в виде последнего четвертого кружка на ромбической диаграмме. Это является вторым шагом. Как видно, второй шаг требует выполнения квадратуры и приводит к появлению свободной постоянной интегрирования в конечных потенциалах $U_{12}, V_{12}, V_{12}^{*}$.

Если мы выполняем указанные два шага для каждой из трех пар $\left\{\omega_{1}, \omega_{2}\right\},\left\{\omega_{1}, \omega_{3}\right\}$, $\left\{\omega_{2}, \omega_{3}\right\}$, показанных на рис. $7 б$ (что дает три дополнительных постоянных интегрирования), алгебраическая формула суперпозиции Бьянки (18) дает решение $\theta^{\prime}$ системы (12) для множества потенциалов $U_{12}, V_{12}, V_{12}^{*}$, полученных на втором шаге.

Формула (18) была доказана в [18] для случая уравнения Мутара $\omega_{x y}=U(x, y) \omega$. Прямое вычисление показывает, что она сохраняет динамику потенциалов, определенную формулой (1), как утверждает теорема 5.

Используя (12) и (18), легко доказать следующие утверждения.

1. Если начать с потенциалов $U_{0}=V_{0}=V^{*}=0$ и выбрать произвольные гармонические многочлены в качестве начальных решений $\omega_{i}, i=1,2, \ldots, N$, то потенциалы, полученные на первых двух шагах, будут рациональными функциями от $(x, y, t)$. Все потенциалы, полученные на последующих шагах, также будут рациональными решениями уравнения $\mathrm{BH}$. 
2. Если нам известно некоторое большое множество решений $\{\psi\}$ уравнения Шредингера $\left(\partial \bar{\partial}+U_{0}\right) \psi=0$ для исходного потенциала $U_{0}$, то с учетом теоремы 5 можно построить соответствующие решения уравнения $\left(\partial \bar{\partial}+U_{12}\right) \tilde{\psi}=0$, используя квадратуры (на втором шаге), если мы положим $\omega_{3}=\psi$ в формулах суперпозиции (12) и (18).

3. Отсюда вытекает, что мы получаем потенциалы $U_{12}$, интегрируемые на нулевом уровне энергии, если начинаем с некоторого интегрируемого потенциала $U_{0}$, например $U_{0}=0$.

\section{6. ЗАМЕЧАНИЕ О ПЕРИОДИЧЕСКИХ И КВАЗИПЕРИОДИЧЕСКИХ СОЛИТОНАХ}

Такая же конструкция может быть применена к случаю, когда исходные функции $\omega_{1}$ и $\omega_{2}$ удовлетворяют уравнению Шредингера с постоянным потенциалом $u=-k^{2}$ :

$$
\left(-\Delta-k^{2}\right) \omega_{1}=\left(-\Delta-k^{2}\right) \omega_{2}=0
$$

Тогда вторая итерация вновь может дать интегрируемый потенциал, который будет гладким и периодическим. Мы приведем здесь такой пример.

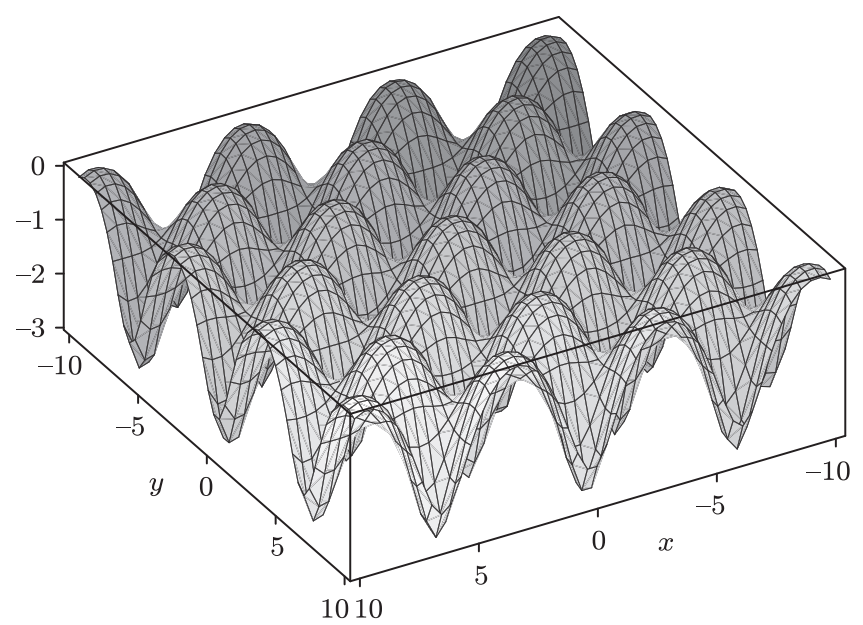

Рис. 8. Периодический потенциал $\tilde{\tilde{u}}$, раздел 6.

Пусть

$$
\omega_{1}=\sin k x, \quad \omega_{2}=\sin (a x+b y), \quad a^{2}+b^{2}=k^{2},
$$

и преобразование Мутара, определенное $\omega_{1}$, отображает $\omega_{2}$ в $\theta_{1}$ (с точностью до слагаемых, кратных $\left.1 / \omega_{1}\right)$ :

$$
\theta_{1}=\frac{b}{2 \sin k x}\left(\frac{\cos (a x+b y+k x)}{a+k}-\frac{\cos (a x+b y-k x)}{a-k}+C\right),
$$




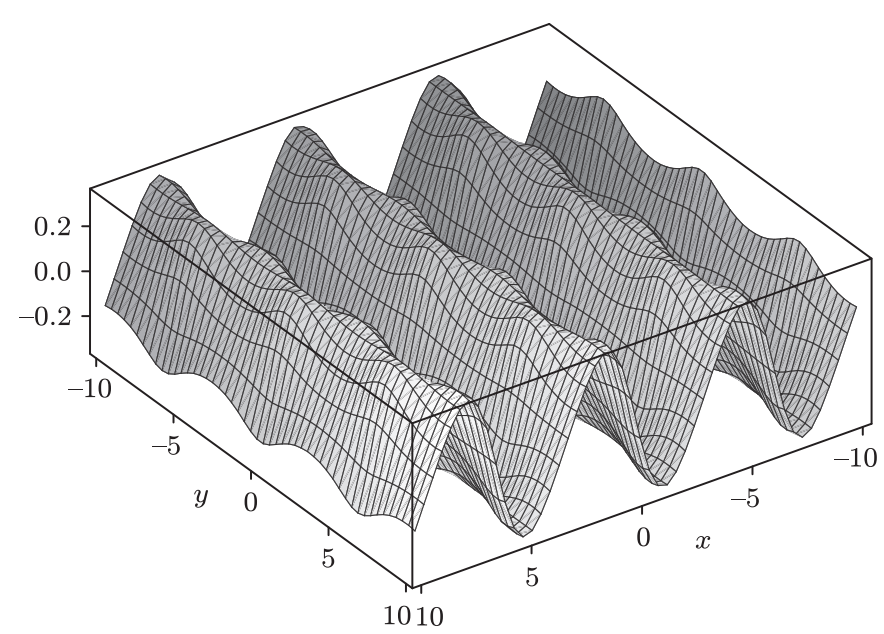

Рис. 9. Решение $\psi_{1}$ для периодического потенциала $\tilde{\tilde{u}}$, раздел 6.

$C=$ const. Новый потенциал равен

$$
\tilde{u}=k^{2}+2 \frac{\cos ^{2} k x}{\sin ^{2} k x} .
$$

Проитерируем преобразование Мутара, используя $\theta_{1}$. В результате получаем следующий новый потенциал:

$$
\tilde{\tilde{u}}=-\tilde{u}+2 \frac{\left(\theta_{1}\right)_{x}^{2}+\left(\theta_{1}\right)_{y}^{2}}{\theta_{1}^{2}}=k^{2}-2 \Delta \ln \left(\omega_{1} \theta_{1}\right),
$$

который будет несингулярен при достаточно большом $C$. Пример подобного периодического потенциала $\tilde{\tilde{u}}$ и соответствующего решения $\psi_{1}=1 / \theta_{1}$ при выборе постоянных $C=3, a=0, b=1, k=1$ показан на рис. 8,9 .

Очевидно, что итерациями преобразования Мутара можно построить интегрируемые двумерные несингулярные периодические и квазипериодические (если $a / b$ не рационально) потенциалы. Полный базис решений для построенных потенциалов $\tilde{\tilde{u}}=-4 U_{12}$ может быть получен применением (18) при $\omega_{3}=e^{i(p x+q y)}, p^{2}+q^{2}=k^{2}$. Для изучения спектральных свойств получаемых потенциалов и динамики соответствующих решений уравнения ВН необходимо отдельное исследование.

Благодарности. Авторы выражают благодарность П. Г. Гриневичу и С. П. Новикову за полезные обсуждения. Настоящая работа поддержана РФФИ (гранты № 06-01-72551 (И. А. Тайманов), № 06-01-00814 (С. П. Царев)). Работа И. А. Тайманова также поддержана Комплексным интеграционным проектом 2.15 СО РАН и Математическим институтом Макса Планка (Бонн).

\section{Список литературы}

[1] T. Moutard, J. École Polyt., 45 (1878), 1-11.

[2] И. А. Тайманов, С. П. Царев, УМН, 62:3(375) (2007), 217-218. 
[3] Л. Д. Фаддеев, “Обратная задача квантовой теории рассеяния. II”, Итоги науки и техники. Соврем. проблемы матем., 3, ред. Р. В. Гамкрелидзе, ВИНИТИ, М., 1974, 93-180.

[4] Р. Г. Новиков, Г. М. Хенкин, УМН, 42:3(255) (1987), 93-152.

[5] А. Н. Веселов, С. П. Новиков, ДАН СССР, 279 (1984), 20-24.

[6] C. E. Kenig, G. Ponce, L. Vega, J. Amer. Math. Soc., 4:2 (1991), 323-347.

[7] В. А. Дубровин, И. М. Кричевер, С. П. Новиков, ДАН СССР, 229 (1976), 15-18.

[8] П. Г. Гриневич, С. В. Манаков, Функи. анализ и его прил., 20:2 (1986), 14-24.

[9] П. Г. Гриневич, С. П. Новиков, Функи. анализ и его прил., 22:1 (1988), 23-33.

[10] G. Darboux, C. R. Acad. Sci. Paris, 94 (1882), 1456-1459.

[11] M. Adler, J. Moser, Comm. Math. Phys., 61:1 (1978), 1-30.

[12] H. Airault, H. P. McKean, J. Moser, Comm. Pure Appl. Math., 30:1 (1977), 95-148.

[13] S. P. Novikov, A. P. Veselov, "Exactly solvable two-dimensional Schrödinger operators and Laplace transformations", Solitons, Geometry, and Topology: On the Crossroad, Amer. Math. Soc. Transl. Ser. 2, 179, eds. V. M. Buchstaber, S. P. Novikov, AMS, Providence, RI, 1997, 109-132.

[14] V. B. Matveev, M. A. Salle, Darboux Transformations and Solitons, Springer Ser. Nonlinear Dynam., Springer, Berlin, 1991.

[15] Heng-Chun Hu, Sen-Yue Lou, Qing-Ping Liu, Chinese Phys. Lett., 20 (2003), 1413-1415.

[16] C. Athorne, J. J. C. Nimmo, Inverse Probl., 7:6 (1991), 809-826.

[17] В. А. Дубровин, С. П. Новиков, ЖЭТФ, 67:6 (1974), 2131-2144.

[18] L. Bianchi, Lezioni di geometria differenziale, V. 1-4, Zanichelli, Bologna, 1923-1927.

[19] Е. И. Ганжа, С. П. Царев, УМН, 51:6(312) (1996), 197-198; arXiv: solv-int/9606003.

Поступила в редакцию 21.01.2008 